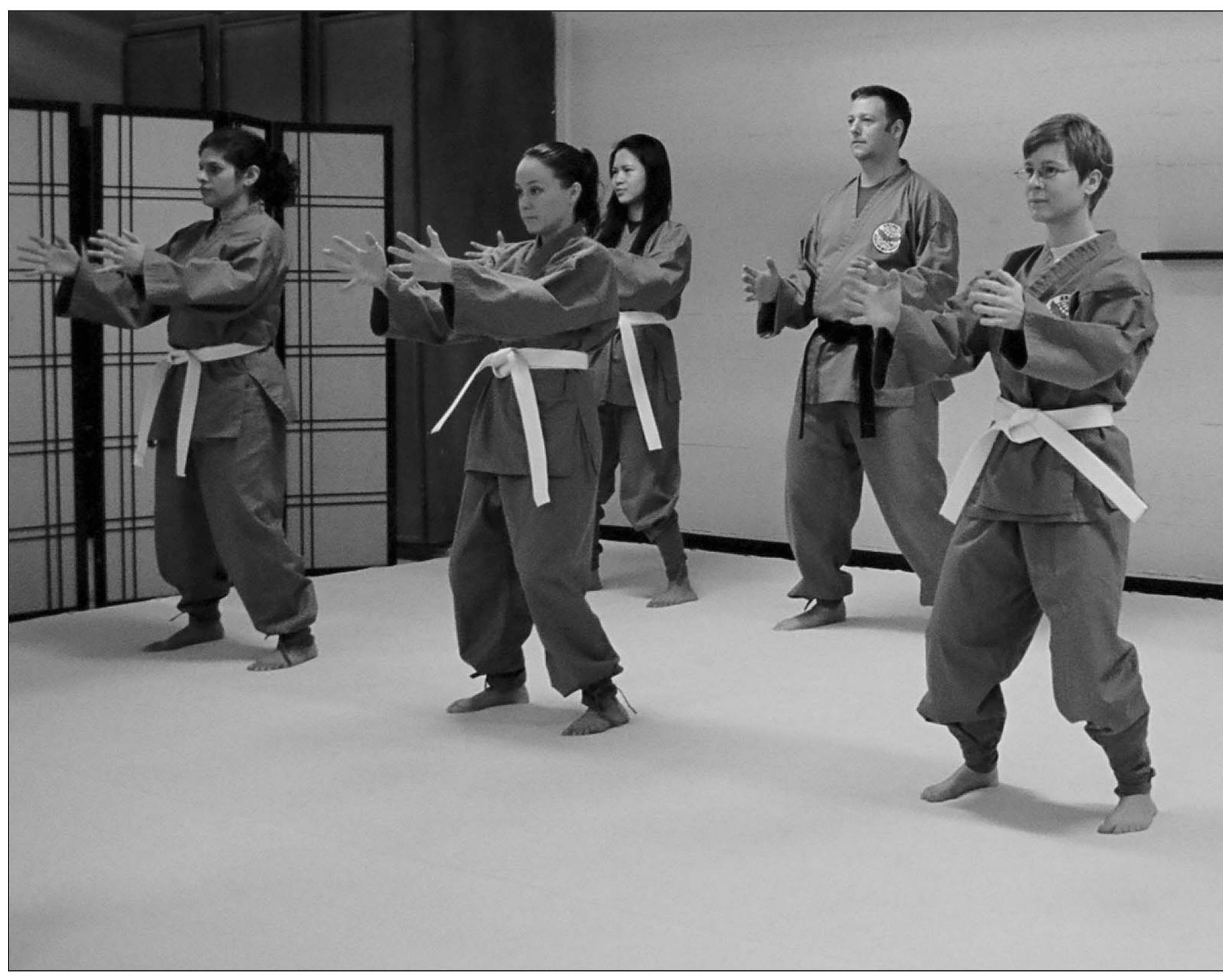

\title{
Cultivando el campo del elixir con la respiración Danjun del Sinmoo Hapkido
}

Sean Bradley 


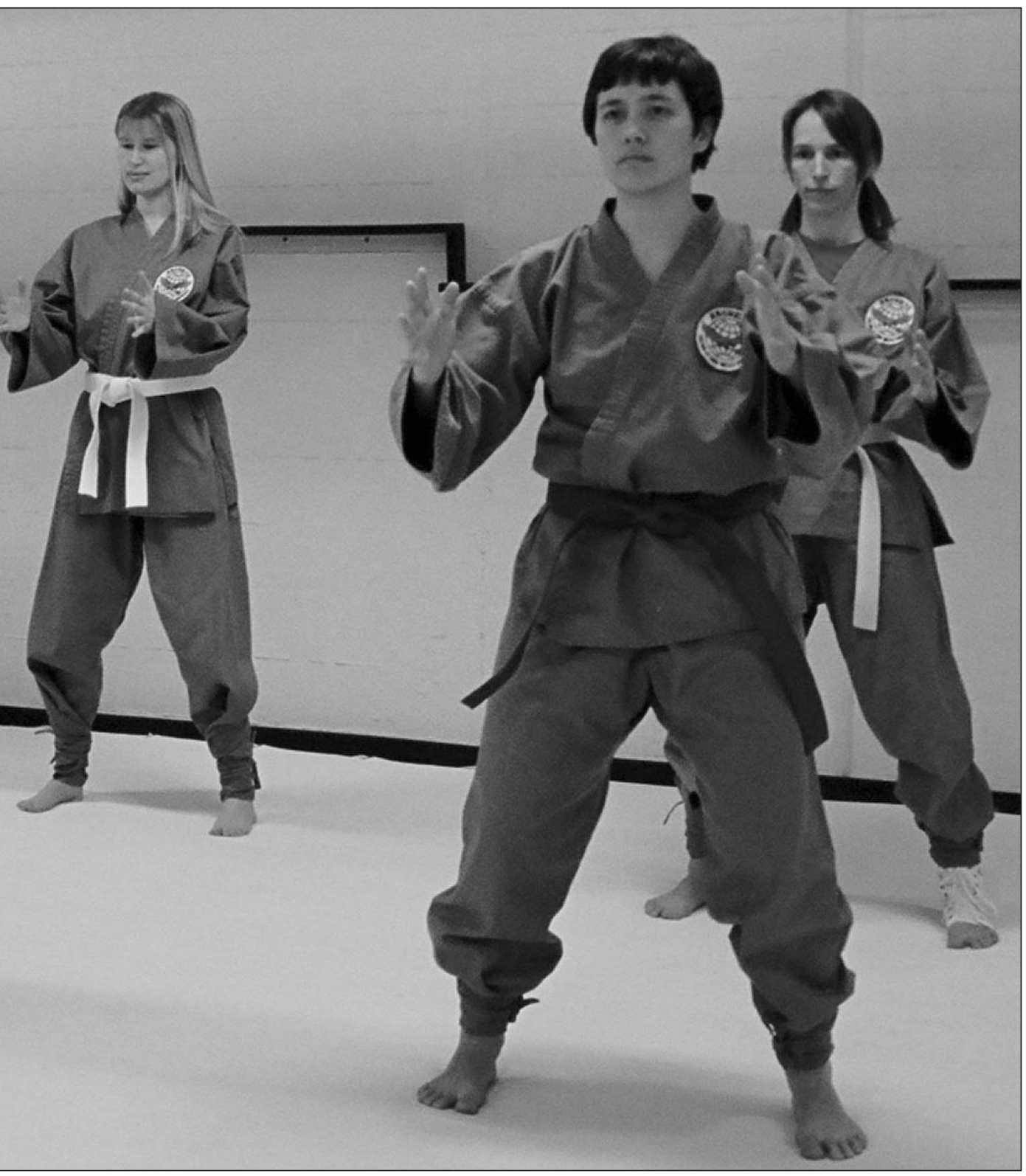

\section{Resumen}

La respiración Danjun es la primera técnica enseñada por el Gran Maestro Ji Han-jae en el arte del Sinmoo Hapkido. Este artículo tratará los aspectos anatómicos y fisiológicos de dicha técnica, así como la teoría que le sirve de fundamento. Utilizando el conocimiento médico occidental, conceptos de acupuntura tradicional, y las enseñanzas de Sinmoo Hapkido, exploraremos las bases del ejercicio, la técnica y preparación correctas, los ciclos de respiración, y las recomendaciones para la práctica. Como ejercicio de respiración básico que implica el movimiento de la energía interna (ki) a través del cuerpo, la respiración danjun se usa como un ejercicio para calentar, como elemento adjunto en varias disciplinas físicas y mentales, así como una ayuda para la salud general. Fácil de aprender, siendo no obstante una técnica efectiva, la respiración danjun puede ser un complemento valioso para cualquier práctica de artes marciales.
Los principiantes practican la respiración Danjun antes del comienzo de la clase.

Fotografías cortesía de Lorene Ledesma, Jeff Hazen, y Suen Sohn. 


\section{Introducción}

En 1984, el Gran Maestro Ji Han-jae creó el Sinmoo Hapkido y presentó su arte al mundo. Aunque no hay traducción inglesa exacta, Sinmoo se traduce simplemente como "arte marcial de mente superior" ( $\sin =$ mente superior; moo $=$ arte marcial). Hapkido significa "el camino de coordinar/armonizar la energía”. Con un fuerte énfasis en el desarrollo mental y espiritual, el Sinmoo Hapkido va más allá de las técnicas físicas básicas del Hapkido coreano tradicional y entra en una esfera de entrenamiento que implica todos los aspectos del yo. Además de las sólidas técnicas físicas presentes en todas las formas de Hapkido, el Sinmoo Hapkido enseña una filosofía espiritual y un amplio repertorio de ejercicios mentales.

Con numerosos ejercicios de respiración y meditación, el Sinmoo Hapkido cambia el foco de atención desde el conglomerado de técnicas externas de golpeo con las piernas y bloqueos de articulaciones por las que es conocido el Hapkido, y lo coloca en el entrenamiento interno y el desarrollo del $\mathrm{ki}^{1}$. El primero de estos ejercicios internos es la respiración danjun (DJB).

El danjun (chino, dantian: dan = cinabrio, tian $=$ campo) es un lugar fisiológico donde se acumula el ki, referido habitualmente como el Campo del Elixir. Aunque no es una estructura anatómica, es una región fisiológica de acumulación y consolidación de la energía. La mayoría de los sistemas de artes marciales reconocen tres danjun en el cuerpo. El primero está situado en el mesencéfalo a nivel de los ojos. El segundo danjun está situado dentro del corazón al nivel del punto de acupuntura Ren-17. El tercero está situado en el abdomen inferior. Los dos primeros tienen que ver con el desarrollo mental y espiritual, y el ki se mueve a estas áreas sólo para su uso inmediato. El ki no se almacena en los danjun superior y medio. El danjun inferior también está implicado en el desarrollo espiritual y mental, pero también se usa para el desarrollo físico. Este es el lugar donde el ki no sólo puede concentrarse y usarse inmediatamente, sino también donde puede ser almacenado y acumulado.

Para los ejercicios básicos como la DJB sólo se utiliza el danjun inferior, por lo que ese ki puede ser acumulado y almacenado para realizar otras actividades o para el ejercicio físico. Los danjun superior y medio son normalmente utilizados sólo cuando hay ya hay una acumulación adecuada de ki en el abdomen inferior. Puesto que el danjun inferior es el único usado en la DJB, a partir de ahora usaré la palabra danjun para referirme únicamente al danjun inferior.

La respiración danjun es uno de los muchos ejercicios encontrados en las artes marciales para el desarrollo del ki. En la misma tradición que el Qigong y el Taijiquan, su fundamento está firmemente enraizado en la Teoría de las Cinco Fases usada en la Medicina Tradicional China (TCM) y acupuntura. La DJB no es simplemente un ejercicio de respiración, como suele pensarse, sino una técnica de meditación profunda que se centra en mover y controlar el ki por dentro del cuerpo. El uso del ki acumulado y controlado depende luego de las necesidades del practicante. Algunos de sus usos comunes son para fortalecer el cuerpo y los órganos, acelerar la curación, agudizar la mente, calentar antes del ejercicio, ayudar en el crecimiento espiritual y ser aplicado físicamente en las situaciones de defensa personal.

\section{Explicación}

El objetivo de la DJB es movilizar y trasladar eficazmente el ki hacia partes específicas del cuerpo. Hacer esto implica generar ki, concentrándolo en un área específica, y luego moverlo al lugar deseado. El ki generado es una 
combinación de constitución hereditaria, ingesta de agua y comida, exposición a la luz del sol, y consumo de oxígeno.

Una vez que generas ki en el cuerpo, lo moverás al danjun. Aquí puedes reunir el ki que está por todo el cuerpo a un punto único para usarlo de forma concentrada. Sólo después de que hayas reunido y acumulado suficiente ki serás capaz de utilizarlo. El lugar exacto de este punto de consolidación del ki en el danjun variará ligeramente de una persona a otra debido a las variables constitucionales de cada individuo. El Gran Maestro Ji señala que es aproximadamente como la anchura de sus dedos índice, corazón y anular. La profundidad es de aproximadamente 2 pulgadas [5,08 $\mathrm{cm}$.] bajo la superficie de la piel en este punto, pero se expande hacia fuera cuanto más ki converge. De acuerdo con Boonchai Apichai, instructor de Qigong en la Universidad de Bastyr, el danjun está a tres dedos de anchura bajo el ombligo, y a tres dedos de anchura hacia el interior del cuerpo. Desde este punto, un radio de tres dedos de anchura se expande para formar una esfera. Esta esfera en el abdomen inferior es el danjun. En la parte anterior, el punto de acupuntura Ren-6, conocido como el "Mar del Qi" corresponde al danjun. Ren-6 esta situado en

\section{Localización del Danjun}

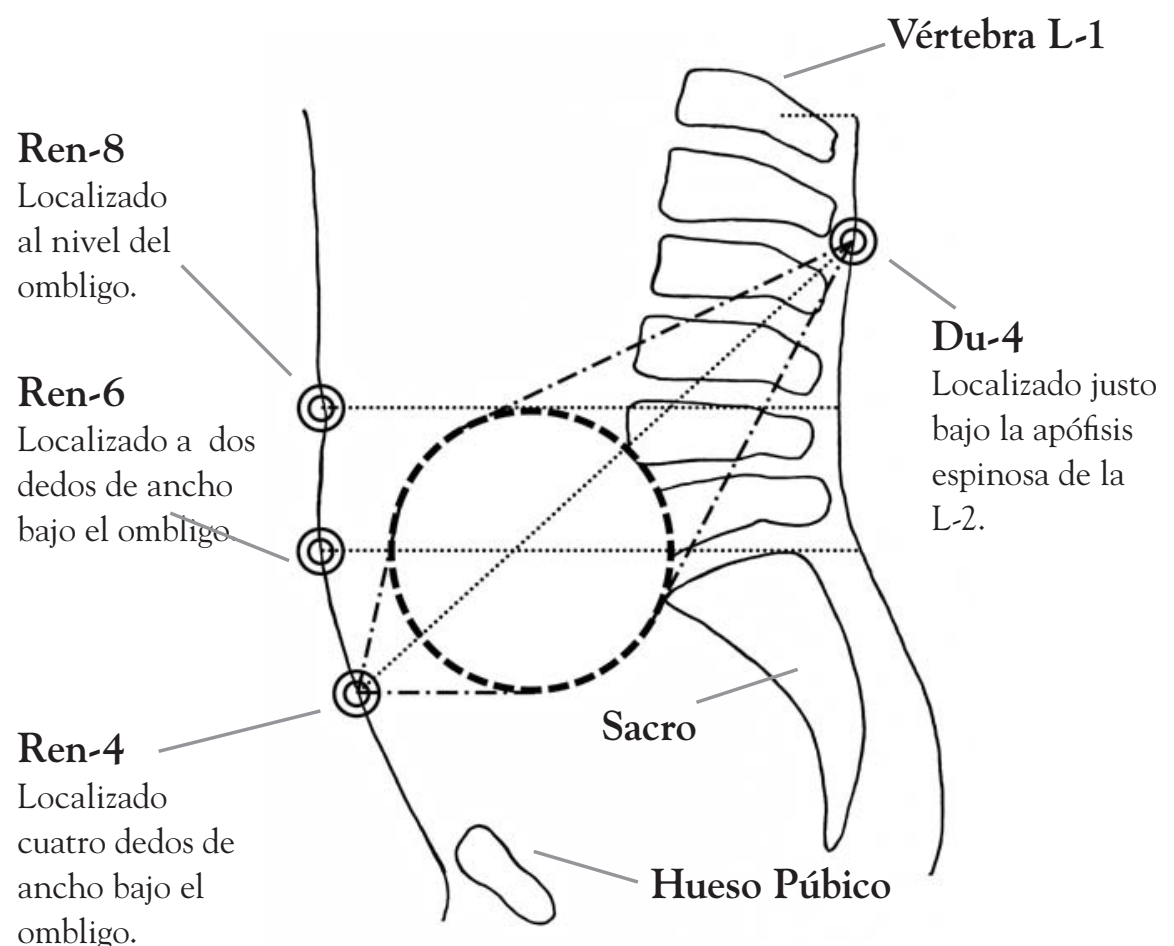

Este diagrama muestra una posible localización del danjun. Esta es el área donde se acumula el ki para usarse por todo el cuerpo. Dependiendo del desarrollo y la salud, el diámetro real puede ser mayor o menor. Usando los meridianos de acupuntura, los puntos Ren en la parte frontal del cuerpo están asociados con el Vaso de la Concepción y el punto Du en la parte posterior del cuerpo con el Vaso Gobernador. El danjun se encuentra en un lugar entre los puntos de acupuntura Du-4 y Ren-4, aproximadamente al nivel de Ren-6. Está situado directamente en la línea media aproximadamente a tres dedos de anchura bajo el ombligo.

Ilustración cortesía de Sean Bradley. 
el vaso Ren o de la Concepción. En la parte posterior, la esfera se extiende a modo de cono hasta un punto justo debajo de la apófisis espinosa de la segunda vértebra lumbar a nivel del $\mathrm{Du}-4$, en el vaso Du o Gobernador.

En Hapkido, el danjun es el punto de acumulación, combinación y propagación del ki. El proceso es el mismo que en los sistemas de acupuntura y qigong, donde se reúne el ki de otras áreas y se acumula en este punto. Desde aquí, podemos utilizar el ki para una amplia variedad de funciones que dependen totalmente del practicante y de sus objetivos en el ejercicio.

\section{Preparación}

Como con cualquier ejercicio de meditación, tu entorno puede influir drásticamente en la efectividad de la técnica. Encontrar el ambiente adecuado puede ser a veces tan importante como la propia técnica.

Un lugar al aire libre, silencioso, que reciba buena luz del sol es el lugar ideal para cualquier técnica de desarrollo del ki. El clima y otros factores ambientales no deberían influir sobre tu entrenamiento, aunque para la mayoría de la gente puede ser demasiada distracción para practicar correctamente la DJB. Si este es el caso, un ambiente interior estable y controlado podría ser preferible. Un lugar interior debería ser silencioso y sin distracciones. Necesita estar bien ventilado, y en el mejor de los casos lo ideal sería conseguir una luz solar adecuada.

Durante un periodo de 24 horas, hay doce horas que son Yin y doce horas que son Yang. A medianoche, la oscuridad, que es el Yin, ha alcanzado su máximo, y comienza a convertirse en luz. A medida que la noche pasa de la oscuridad a la luz del amanecer, el Yang entra en funcionamiento. De esta manera, el periodo del Yang comienza a medianoche y se prolonga hasta el mediodía. De las 5 a las 7 de la mañana es el momento Yang más alto del día. Alcanzará su pico en este momento, y luego disminuirá gradualmente hasta el mediodía, cuando el Yin comenzará de nuevo a entrar en funcionamiento. Este ciclo se repite cada día.

Hay tres momentos al día que el Gran Maestro Ji recomienda para practicar la DJB de manera regular. Antes del amanecer, a mediodía cuando el sol está en su punto más alto, y la puesta de sol son momentos importantes de energía que son perfectos para movilizar el ki dentro de tu cuerpo.

Cuando el Yang alcanza su pico en la mañana, deberías ejercitarte. Este es el momento en el cual el cuerpo tiene la mayor energía para la actividad y el movimiento. Realiza la DJB antes de hacer ejercicio para crear ki con el fin de calentar el cuerpo y usarla para la actividad física. El calentamiento completo del cuerpo que tiene lugar en la DJB te ayudará a estar mejor preparado para un entrenamiento tanto mental como físico.

Desde las 11 de la mañana hasta la 1 de la tarde el Yang está cambiando a Yin. Este es un momento para relajarse y permitir al cuerpo una lenta transición. Practica la DJB en este momento para crear el ki para la curación y la calma. De ser posible, deberías realizar este ejercicio justo antes de realizar una corta siesta.

Por la tarde, de 5 a 7, el Yin está en su punto más alto. Este es el momento en el que el cuerpo se encuentra mejor preparado para descansar y digerir la comida. Practica la DJB en este momento con el fin de crear el ki para fortalecer los órganos antes de cenar.

En relación con el momento del día, es importante tener el sol a tu espalda mientras practicas la DJB, especialmente cuando prácticas al aire libre. La parte de atrás del cuerpo es el lado Yang, y mientras que nuestra espalda está hacia el sol, nuestro "cuerpo se carga" de la misma manera que un panel solar se carga por la acción del sol. El efecto contrario ocurre cuando miras 
de frente, o el lado Yin del cuerpo, hacia el sol. Esto tenderá a debilitar tu fuerza y los beneficios de la DJB no se producirán.

La altitud es otra consideración importante, ya que es necesario tener en cuenta la habilidad de tu cuerpo para absorber y captar oxígeno de manera efectiva. El Gran Maestro Ji recomienda realizar la DJB entre las altitudes de 2000 y 6000 pies [609,6 y 1828,8 m. respectivamente] para colocar al cuerpo en la situación óptima para la meditación espiritual. En cualquier altitud superior a los 6000 pies, el individuo no aclimatado puede correr el peligro de falta de oxígeno.

\section{Posición del cuerpo}

Con la cabeza y los ojos directamente hacia delante, colócate con los pies ligeramente más separados que la anchura de los hombros. Tu espalda debería estar recta, con los hombros ligeramente echados hacia atrás para compensar la frecuente tendencia de girar los hombros hacia delante y arquear
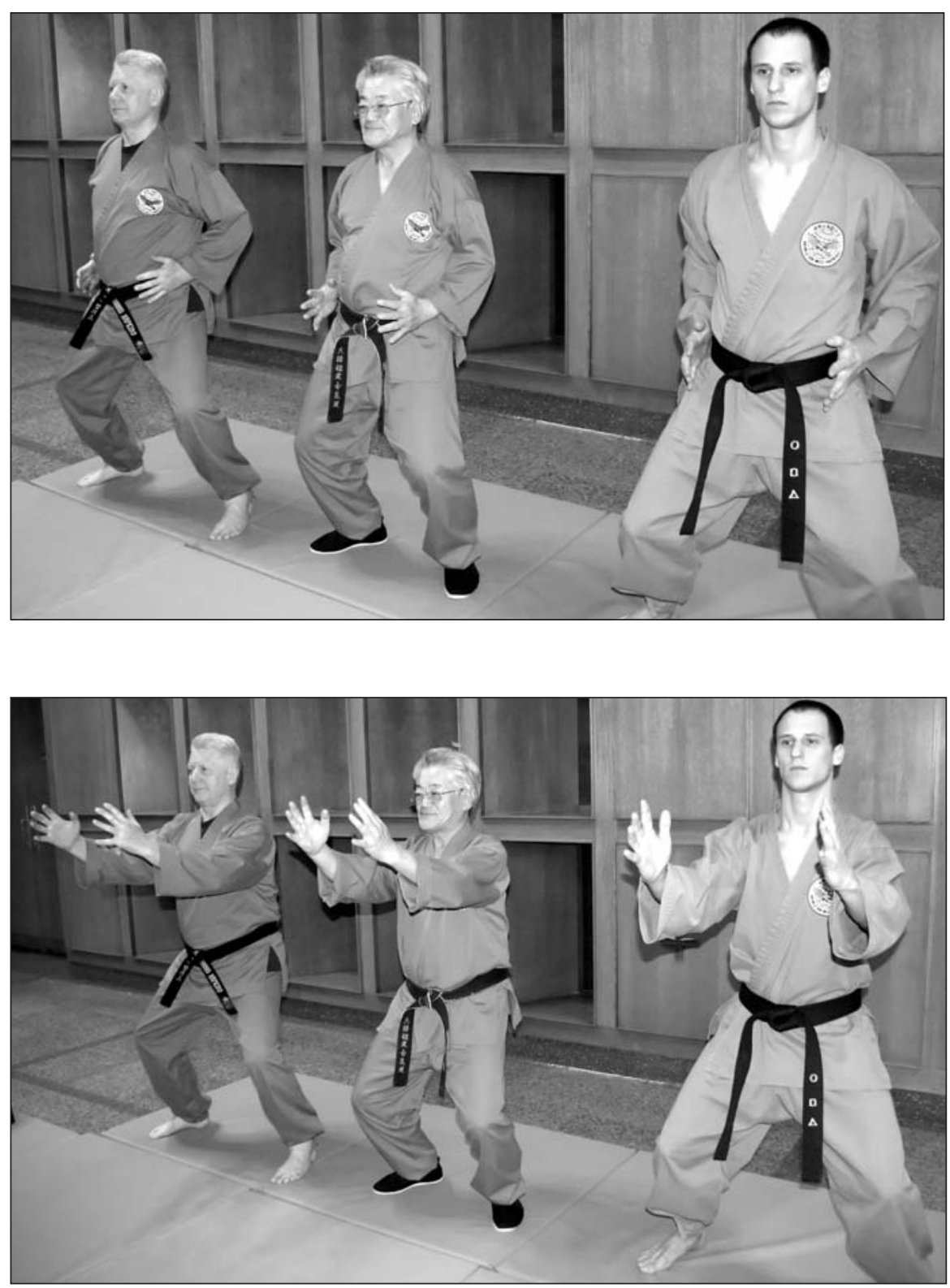

Vista frontal del Maestro Egil Fosslien, el Gran Maestro Ji Han-jae, y el Maestro Sean Bradley practicando la Respiración Danjun. 
la espalda. Los brazos tendrán una ligera flexión de aproximadamente 130 grados a nivel del codo.

Mantén tu cabeza en equilibrio en los tres planos. El sistema vestibular que controla la estabilidad y el equilibrio está situado dentro del oído interno. Los canales semicirculares, el utrículo y el sáculo componen el sistema vestibular, y están revestidos de pelos y llenos de un fluido llamado endolinfa. Cuando tu cuerpo se mueve, el fluido se mueve en distintas direcciones causando que los pelos se inclinen. Cuando los pelos se inclinan generan un impulso nervioso o potencial de acción que va hacia el cerebro, donde posteriormente se interpreta el movimiento.

El utrículo y el sáculo son los órganos otolíticos que se encuentran dentro del oído y miden la aceleración lineal (i.e. anterior/posterior y elevación/depresión). El utrículo mide la inclinación de la cabeza así como el movimiento antero-posterior y debería permanecer inmóvil durante la DJB. El sáculo mide el ascenso y descenso y es la única parte del sistema vestibular que debería moverse durante la DJB. Habrá un ligero ascenso y caída cuando tus rodillas se flexionen y extiendan.

Los canales semicirculares miden la aceleración angular en los tres planos. Los pilotos llaman a estos tres planos inclinación, balanceo y viraje. La inclinación es el movimiento de flexión y extensión -asintiendo con tu cabeza "sí" o arriba y abajo-. El mantener tu nariz y tu mentón en línea prevendrá el cabeceo. Tu nariz y mentón deberían formar una línea que, si se extendiese desde la punta de tu nariz hacia abajo, llegaría perpendicularmente al suelo. Un error frecuente en la práctica de la DJB es tener la cabeza inclinada hacia atrás, levantando ligeramente tanto la nariz como el mentón. El balanceo es la rotación lateral o inclinación lateral. Este es el movimiento por el que llevas tu oreja hacia tu hombro. Para evitar esto en la DJB, tus orejas deberían formar una línea paralela con el suelo.

El equilibrio de la cabeza es importante para conseguir un flujo óptimo de ki por el cuerpo.

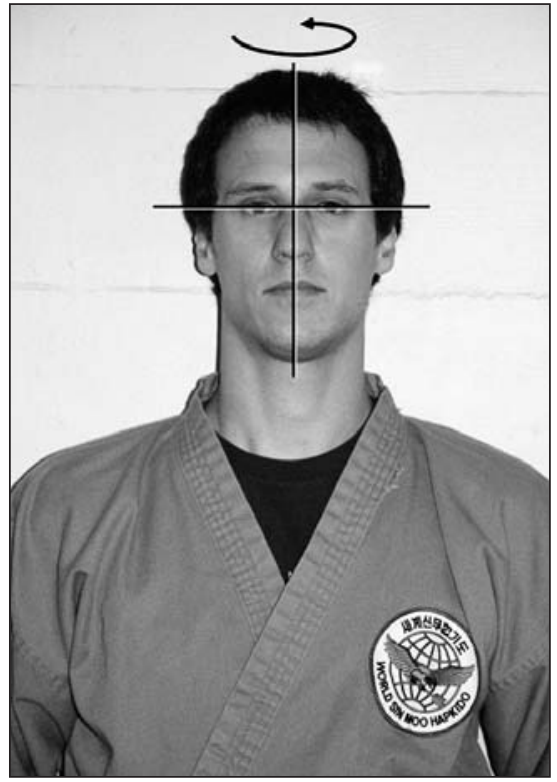

Viraje - equilibrio ocular.

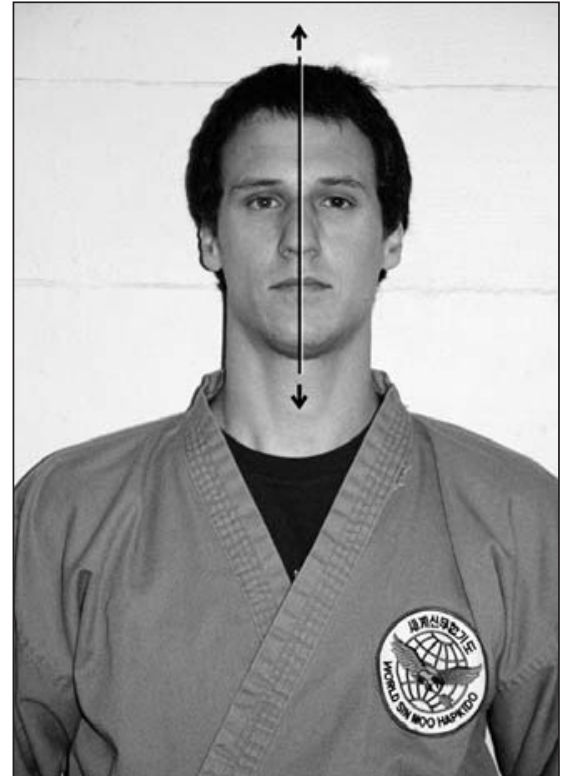

Inclinación - equilibrio de nariz y mentón.

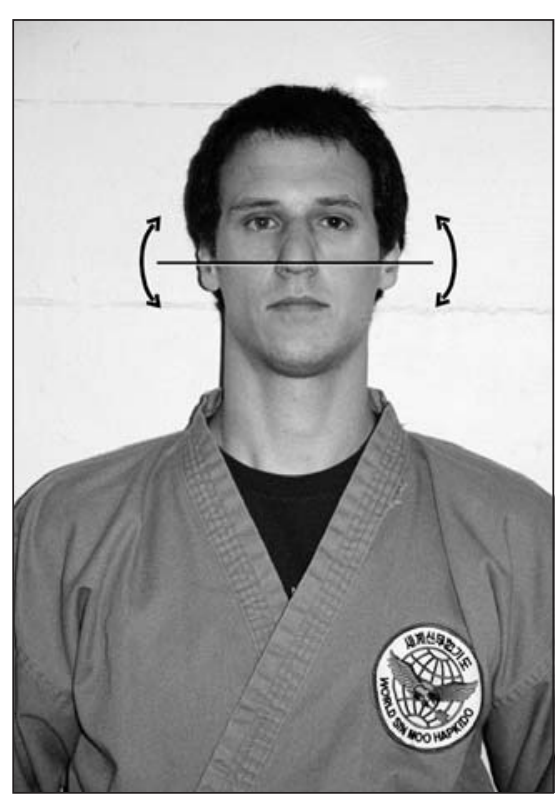

Balanceo - equilibrio del oído 
El viraje es la rotación en el plano horizontal, o mover tu cabeza diciendo "no". La mayoría de la rotación tiene lugar en la articulación atlantoaxial donde la columna vertebral, a nivel del atlas, la primera vértebra cervical (C1), se asienta sobre la segunda vértebra cervical (C2), el axis. El atlas se asienta encima y gira alrededor de la prominencia ósea en el eje conocido como apófisis odontoides. Para prevenir el viraje, tus ojos deberían permanecer fijos hacia el frente a igual distancia de la línea media. Una línea debería ascender directamente por tu columna vertebral, y si continuase debería pasar a mitad de camino entre tus ojos.

Cualquier ligero movimiento en cualquiera de los planos puede causar que "pierdas tu equilibrio", y tu cerebro tendrá que reaccionar para recobrarlo. Aunque esto puede ser muy sutil, incluso los más pequeños movimientos de cabeza pueden provocar que pierdas continuamente el equilibrio. Aunque no te des cuenta de ningún movimiento, si tu cerebro está continuamente trabajando para mantener el equilibrio no eres capaz de trabajar en el desarrollo del ki, ya que tu mente está constántemente perdiendo su atención.

\section{Respiración}

Respira por tu nariz en la DJB. Esto permite a los pequeños pelos nasales y al revestimiento de mucosa filtrar y calentar el aire antes de que entre en los bronquios y pulmones. La boca no hace tan buen trabajo de limpieza del aire ya que carece de este mecanismo de filtrado. Respirar sólo por la nariz prevendrá que muchos alérgenos aéreos, toxinas y otras partículas entren en las vías respiratorias.

El ciclo respiratorio para la DJB es como sigue: inhala durante 8 segundos, mantén durante 8 segundos, presiona durante 4 segundos, y exhala durante 8 segundos. La inspiración larga permite el suficiente calentamiento o enfriamiento del aire por lo que la vía respiratoria no sufre un choque por un cambio drástico de la temperatura. Los ciclos de mantenimiento y presión permiten empujar y mover el ki a diferentes partes del cuerpo. La espiración lenta y regulada permite un mejor control y el fortalecimiento del cuerpo.

En la inspiración normal suave, lo que el Gran Maestro Ji llama, "respiración del bajo estómago", el diafragma se contrae y se mueve inferiormente y se aplana. Cuando el diafragma se expande hacia abajo, la cavidad torácica se amplia. Esto provoca que la presión en los pulmones baje y que el aire exterior entre.

Cuanto más larga y lenta sea la respiración, más hinchados están los pulmones. Cuando el aire entra, los alvéolos o sacos alveolares que forman los pulmones se llenan de aire. Cuando se llenan, se expanden y estiran. La inhalación lenta no sólo permite que más alvéolos se llenen de aire, sino que les permitirá hacerlo de una manera más controlada. Este lento estiramiento provocará menos tensión en las células epiteliales que recubren el interior de los alvéolos.

Además de la salud pulmonar, esta profunda contracción tiende a fortalecer el diafragma y aumenta su eficacia. El Sinmoo Hapkido postula que, debido a que el diafragma es un músculo esquelético como el bíceps braquial, la contracción lenta y controlada incrementará su fuerza y resistencia de la misma manera que el entrenamiento de pesas puede beneficiar a los brazos. En la inspiración suave y lenta, también usamos los músculos intercostales externos que unen las costillas. Ayudan a expandir el tórax en sentido lateral y antero-posterior al elevar la caja torácica y subir el esternón. Cuanto más eficiente es el diafragma, menor es el uso de estos músculos y menos obvia llega a ser la respiración. 
La espiración suave no debería utilizar activamente ninguna tensión intencionada de los músculos del estómago, sino que sólo debería ser un retroceso natural de los pulmones. Los sacos alveolares que forman el tejido pulmonar son estructuras elásticas muy parecidas a un globo. Una vez que los pulmones están llenos, los alvéolos retrocederán, volviendo a su posición relajada y empujando el aire fuera de los pulmones.

La espiración controlada, sin embargo, usa el diafragma y los músculos abdominales y permite un retroceso lento y limitado. Usando esta respiración lenta y controlada fortaleces el diafragma al no permitirle que vuelva bruscamente a su lugar, sino que ejerces una tensión negativa en la fase de relajación. También fortaleces los músculos abdominales al tensarlos y contraerlos cuando exhalas. El tener unos músculos abdominales más fuertes y más tonificados dará una mejor protección a los órganos internos, al danjun, y al plexo solar.

\section{Ciclos}

Hay ocho ciclos de respiración en la DJB. Esto se relaciona directamente con la teoría de las cinco fases en la Medicina Tradicional China (TCM). Las cinco fases o elementos en la TCM son madera, fuego, tierra, metal, y

$\begin{array}{llllll}\text { Elemento } & \text { Órgano Zang } & \text { Órgano Fu } & \text { Color } & \text { Tejido } & \text { Emoción } \\ \text { Fuego } & \text { Corazón } & \text { Intestino delgado } & \text { Rojo } & \text { Sanguíneo } & \text { Alegría } \\ \text { Metal } & \text { Pulmón } & \text { Intestino grueso } & \text { Blanco } & \text { Piel/pelo } & \text { Tristeza } \\ \text { Madera } & \text { Hígado } & \text { Vesícula biliar } & \text { Verde/azul } & \text { Tendón } & \text { Ira } \\ \text { Tierra } & \text { Bazo } & \text { Estómago } & \text { Amarillo } & \text { Músculo } & \text { Preocupación } \\ \text { Agua } & \text { Riñón } & \text { Vejiga } & \text { Negro } & \text { Hueso } & \text { Miedo }\end{array}$

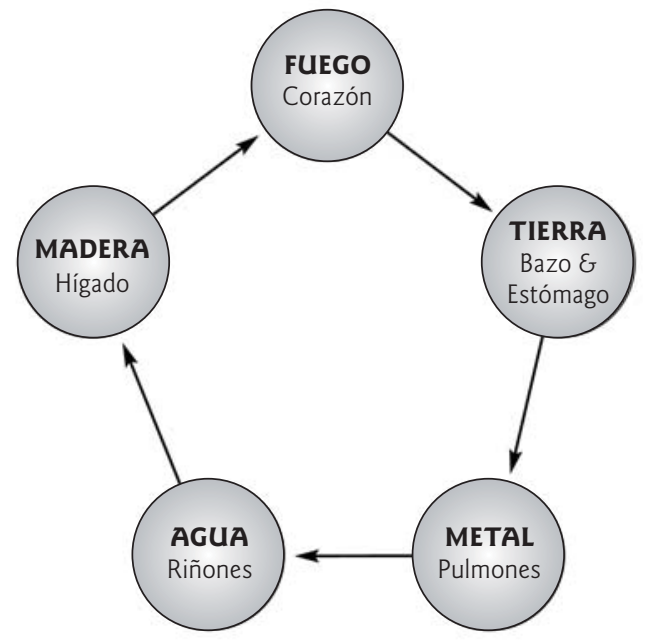

El ciclo generador

Este ciclo es para el desarrollo del ki en el cuerpo. No se usa en la respiración danjun, pero es parte del ciclo diario de respiración que sucede por sí mismo.

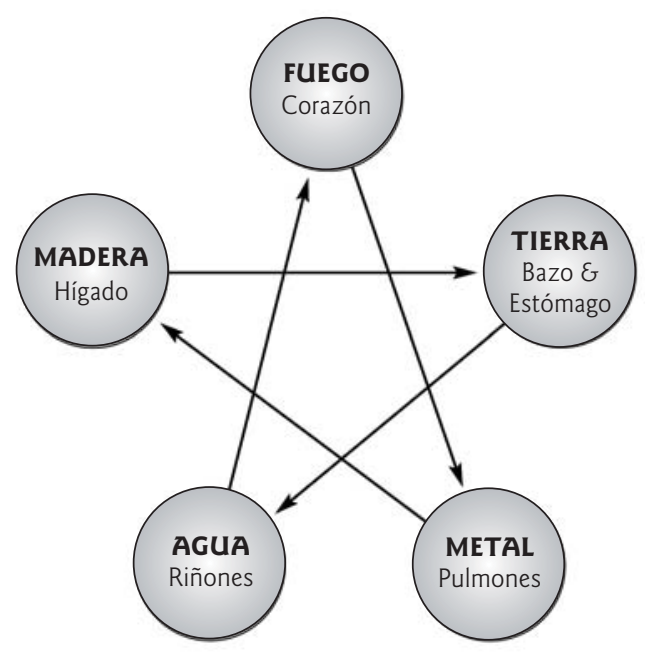

El ciclo controlador

Este es el ciclo que se sigue en la respiración danjun, donde el practicante comienza dirigiendo el ki hacia el lado izquierdo del corazón y termina en el riñón derecho. 
agua. Cada uno de estos elementos se relaciona con dos órganos principales del cuerpo. Para cada elemento hay un órgano Zang y un órgano Fu. Los órganos Zang se relacionan con el Yin y los órganos Fu están asociados con el Yang. Los cinco órganos Zang primarios son los órganos sólidos que son responsables de almacenar el ki puro. Estos órganos son hígado, corazón, bazo, pulmones, y riñones. Los cinco órganos $\mathrm{Fu}^{2}$ son responsables de transmitir el agua y la comida sin almacenarlos. Los órganos Fu son intestino delgado, intestino grueso, vesícula biliar, estómago, y vejiga urinaria.

Los cinco elementos se generan unos a los otros en un ciclo generador o inter-organizado. La madera puede arder, dando fuego. El fuego crea ceniza, que es tierra. Los procesos de la tierra crean varios metales. Cuando fundes varios metales, creas vapor o agua. Las plantas necesitan agua para crecer y producir madera. Más que en el ciclo generador, la DJB se centra en el ciclo interactivo o controlador. En este ciclo, el fuego puede moldear el metal, el metal puede cortar la madera, la madera mantiene la tierra en su lugar, la tierra desvía y contiene el agua, y el agua apaga el fuego. Nuestro cuerpo toma el control de la parte generadora del ciclo, pero, a través de la DJB, podemos controlar el ki que desarrolla, y usarla para lo que lo necesitemos.

En resumen, el ciclo de las ocho respiraciones incluye la dirección del ki hacia el corazón, pulmones, hígado, estómago/bazo, y riñones. Practicar la DJB en este orden ayuda a controlar el ki que nuestro cuerpo está creando y lo dirige a ciertas áreas que lo necesitan.

La razón para hacer ocho ciclos en vez de sólo cinco se debe a los órganos pares. Para el corazón hay dos ciclos debido a la implicación del lado izquierdo y derecho en la circulación sistémica y pulmonar. Hay dos ciclos para los pulmones y dos ciclos para los riñones debido a que hay dos órganos. De este modo, el ciclo entero se compone de dos respiraciones para el corazón, una respiración para cada pulmón, una respiración para el hígado, una respiración para el combinado estómago/bazo, y una respiración para cada riñón.

En los ciclos en que el órgano es par, es importante comenzar en el lado izquierdo. Esto tiene que ver con las cámaras del corazón. En el lado izquierdo del corazón hay sangre oxigenada de los pulmones que está alimentando al cuerpo. En el lado derecho del corazón hay sangre poco oxigenada que necesita ir a los pulmones para obtener más oxígeno. Empezando en el lado izquierdo cuando se hace este ejercicio, permitimos a este nutriente rellenar nuestro cuerpo para después crear. Empezar en el lado derecho podría ser potencialmente peligroso puesto que sin la energía de la sangre oxigenada nos arriesgamos a no tener suficiente energía para realizar adecuadamente el ejercicio e incluso puede causar más daños.

Más importante que el flujo sanguíneo es la relación entre el Yin y el Yang. El lado izquierdo del cuerpo es el lado Yang y el derecho el Yin. Del mismo modo que necesitas una cerilla para encender un carbón empapado en combustible, el Yang es necesario para poner en marcha el Yin. El Yang, siendo el lado activo, necesita continuar hacia el lado Yin pasivo, y por lo tanto está primero en los ciclos respiratorios. El Gran Maestro Ji se refiere a esto enseñando a usar el lado izquierdo para generar y el lado derecho para la aplicación.

\section{Técnica}

Comenzando en una posición relajada, inhala lentamente por tu nariz durante ocho segundos. Cuando inhales por tu nariz, cierra tu boca y presiona tu lengua contra el paladar. Haciendo esto se une el Vaso Ren o Concepción que sube por la parte frontal del cuerpo y termina en la punta de la lengua, con el Vaso Du o Gobernador que sube por el dorso del cuerpo, por encima

\section{Órganos Zhang internos}

1. Lado izquierdo del corazón.

2. Lado derecho del corazón.

3. Pulmón izquierdo.

4. Pulmón derecho.

5. Hígado.

6. Bazo (estómago).

7. Riñón izquierdo.

8. Riñón derecho.

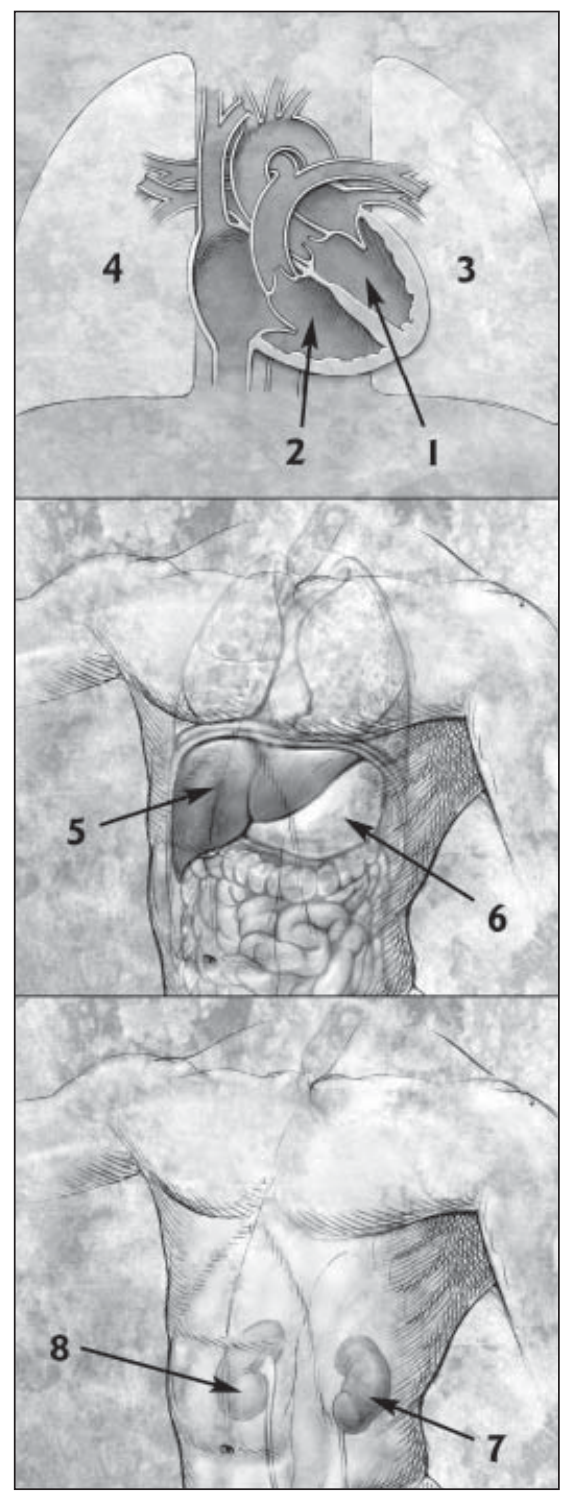

En la respiración danjun, ocho ciclos de respiración constituyen un ejercicio completo. Cada respiración corresponde a uno de los ocho órganos. 


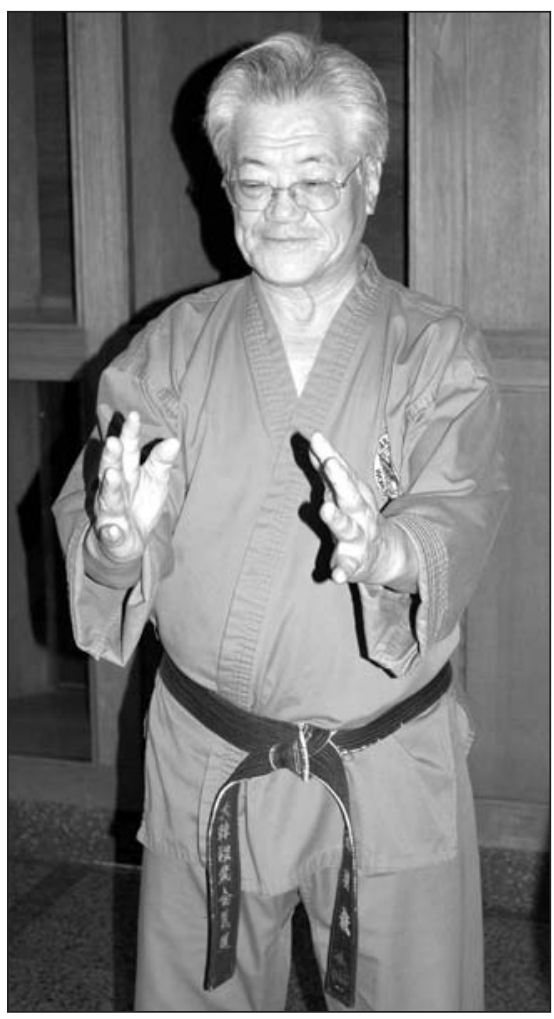

El Gran Maestro Ji Han-jae demuestra la posición correcta de la "Mano Viva".

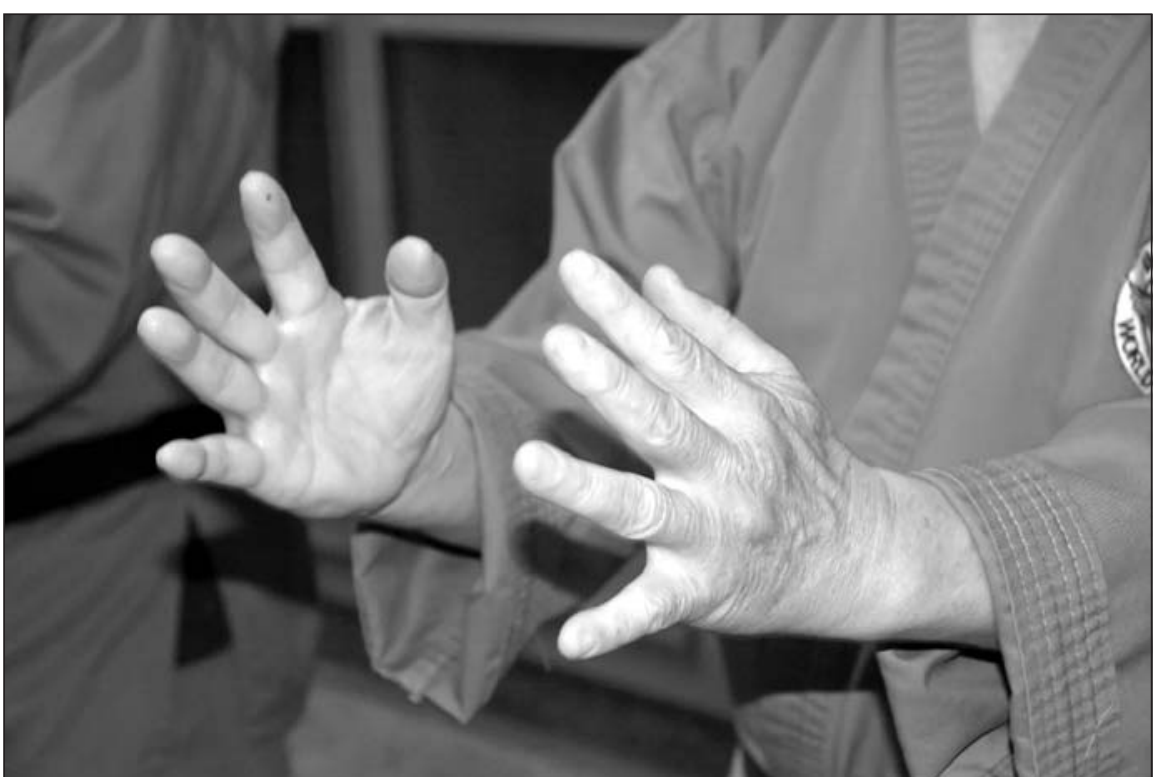

de la cabeza, y termina en el paladar. Esto completa un ciclo meridiano conocido como el ciclo microcósmico que permite un flujo constante y eficiente del ki por el centro del cuerpo. Asegúrate de mantener este contacto de la lengua durante todo el ejercicio. Inspira lentamente, asegurándote de no calentar o enfriar el aire. Respira profundamente e imagina que dejas fluir el aire hacia tu danjun, y no simplemente a tu pecho.

Al final de la inhalación, relájate sobre tus pies y lleva tus manos, con las palmas una frente a otra y los dedos extendidos, a nivel de tus caderas aproximadamente a una pulgada $[2,54 \mathrm{~cm}]$ de tu cuerpo. Tus manos debe-
Vista lateral del movimiento del cuerpo durante la Respiración Danjun.

- Inspirando (1-2)

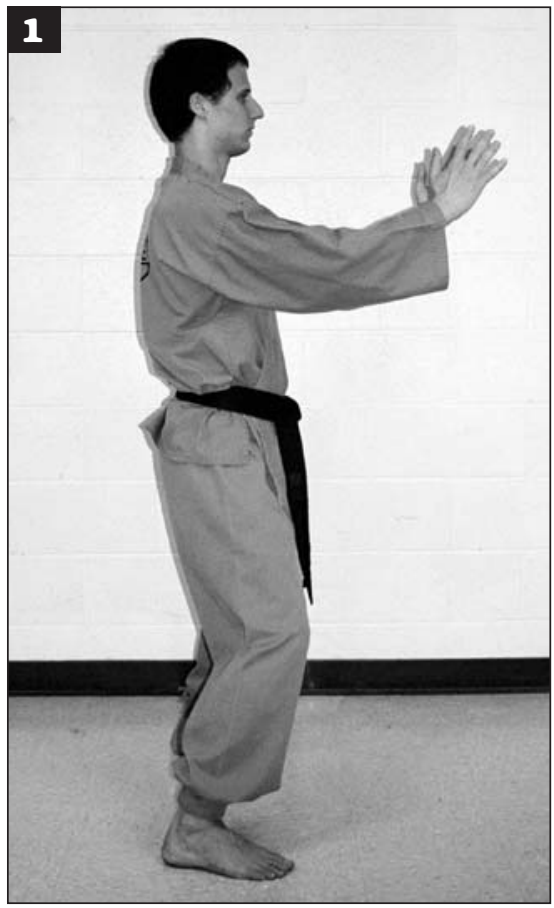

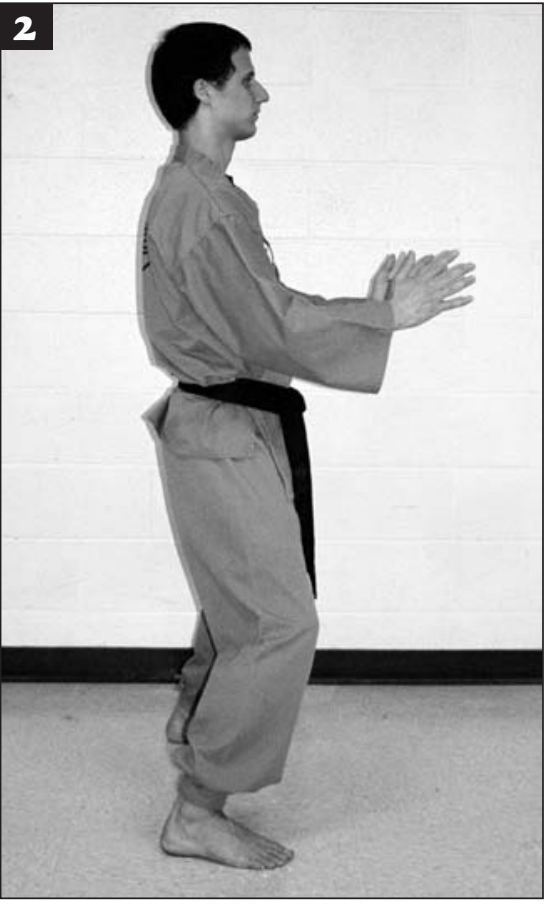

- Mantener y presionar (3)

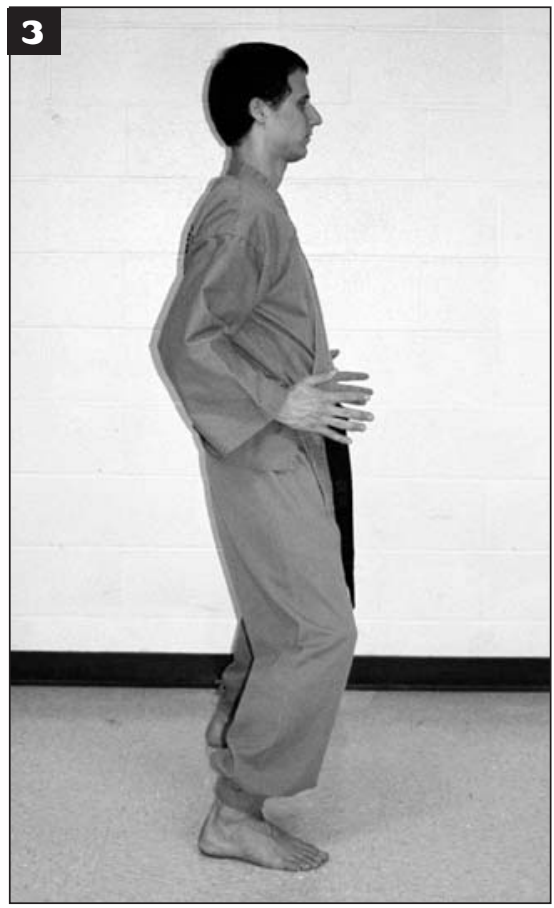


rían estar en la posición "Manos vivas" o "Manos Llenas de ki". Extiende los dedos y el pulgar. Dobla tus nudillos ligeramente mientras mantienes tus palmas en una posición paralela. Manteniendo tu rostro tranquilo y relajado, mantén tu respiración durante ocho segundos. Cuando haces esto, dirige el ki que acabas de generar (introduciendo el oxígeno dentro del cuerpo) hacia el área del danjun.

Cuando la fase de mantenimiento se ha completado, comienza la fase de presión. Aprieta tus muelas con fuerza, y presiona como si estuvieras haciendo de vientre, comprimiendo tu esfínter anal cerrado. Intenta girar tus pies hacia el interior mientras te contraes contra el suelo y tensas tus piernas. Aprieta los omóplatos a la vez, utilizando los músculos de la espalda para apretar tus brazos contra el cuerpo mientras contraes los bíceps y antebrazos para mantenerlos en su lugar. Todas estas contracciones deberían ser isométricas y tu cuerpo no se debería mover. Cuando presionas durante cuatro segundos visualiza el ki que has acumulado dentro del danjun comprimiéndolo en una pequeña bola. Convierte el ki, ahora una mancha difusa de energía, en una pequeña bola de energía concentrada del tamaño de una canica.

Espira lentamente durante ocho segundos por tu nariz. Mantén la boca apretada y el cuerpo tenso. Lentamente levanta tus manos del nivel de la cadera pivotando en tus hombros para alzar tus brazos hasta que las puntas de tus dedos están al nivel de los ojos. No dobles más tus brazos, mantén la ligera flexión de 130 grados con la que comenzaste. Mientras levantas tus brazos, visualiza el traslado del ki desde el danjun, dirigiéndolo a ciertas partes de tu cuerpo. Al principio querrás ejercitarte dirigiendo el ki a cada uno de los correspondientes órganos durante los ocho ciclos para fortalecer cada uno de estos órganos. Con mayor experiencia, dirigir el ki hacia las manos es un modo eficaz de generar fuerza para golpes, escapes y para la curación. Para tus propias lesiones, puedes dirigir el ki concentrado al área necesitada con el fin de ayudar a recuperarte mejor y más rápidamente.

- Espirando (3-5).
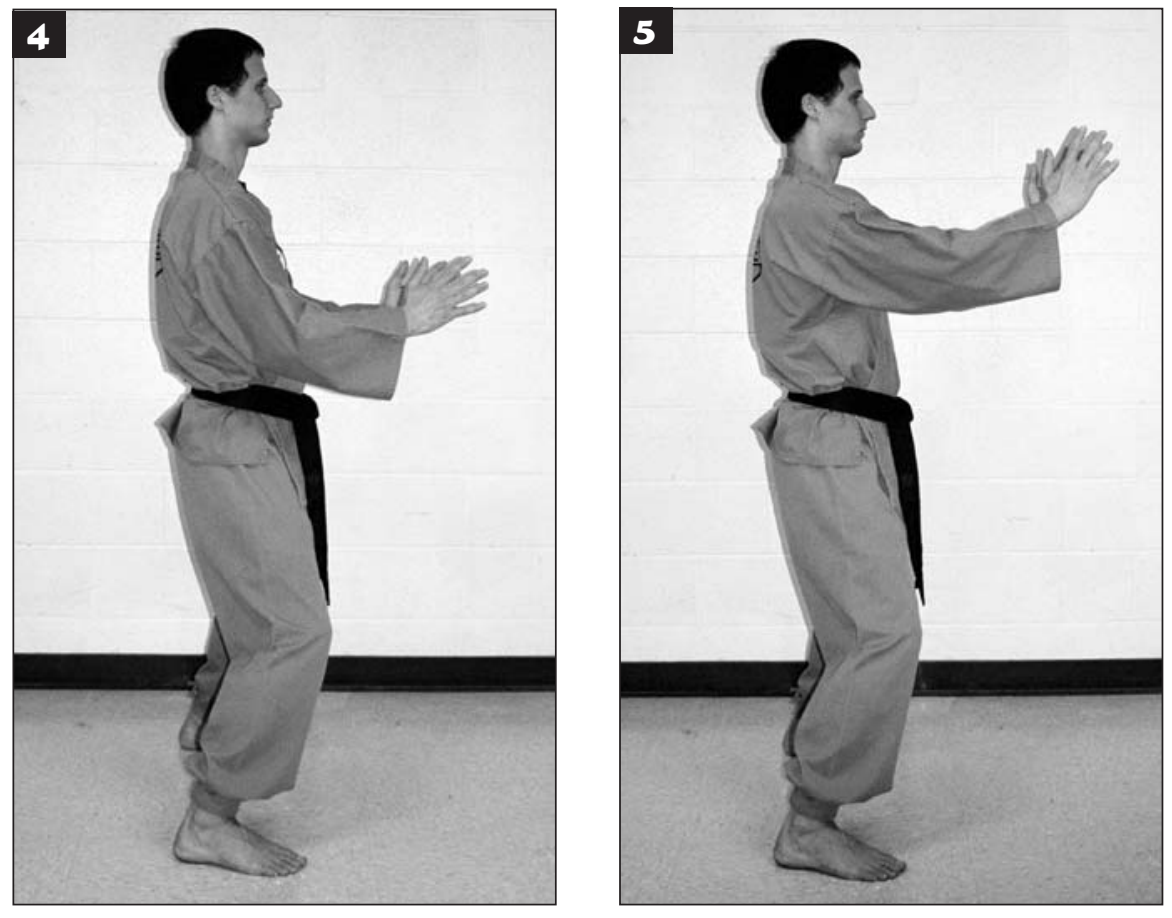
1-A-B) El Gran Maestro Ji Han-jae demuestra la aplicación de la "Mano Viva" realizando una palanca al brazo.
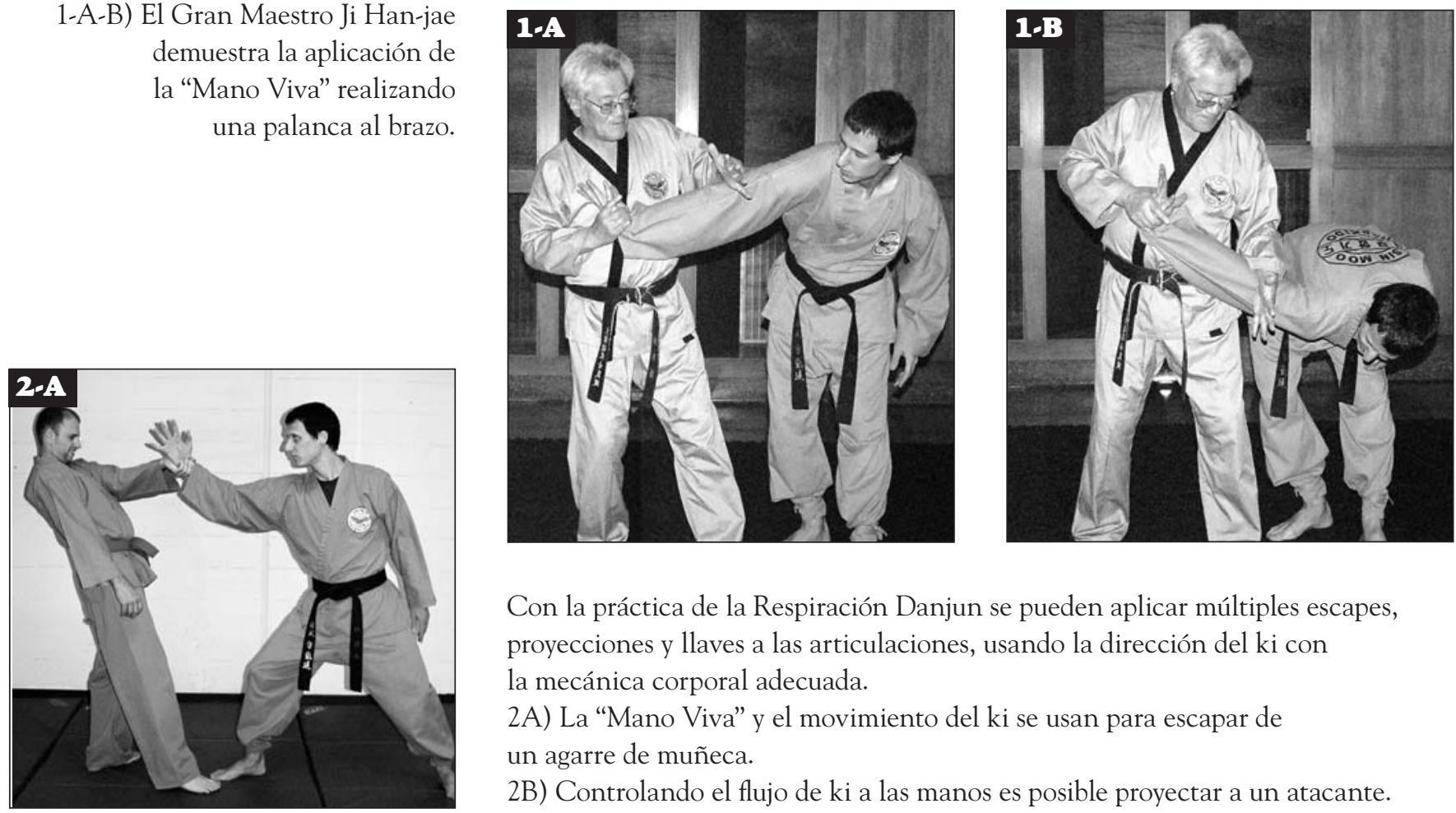

Con la práctica de la Respiración Danjun se pueden aplicar múltiples escapes, proyecciones y llaves a las articulaciones, usando la dirección del ki con la mecánica corporal adecuada.

2A) La "Mano Viva" y el movimiento del ki se usan para escapar de un agarre de muñeca.

2B) Controlando el flujo de ki a las manos es posible proyectar a un atacante.

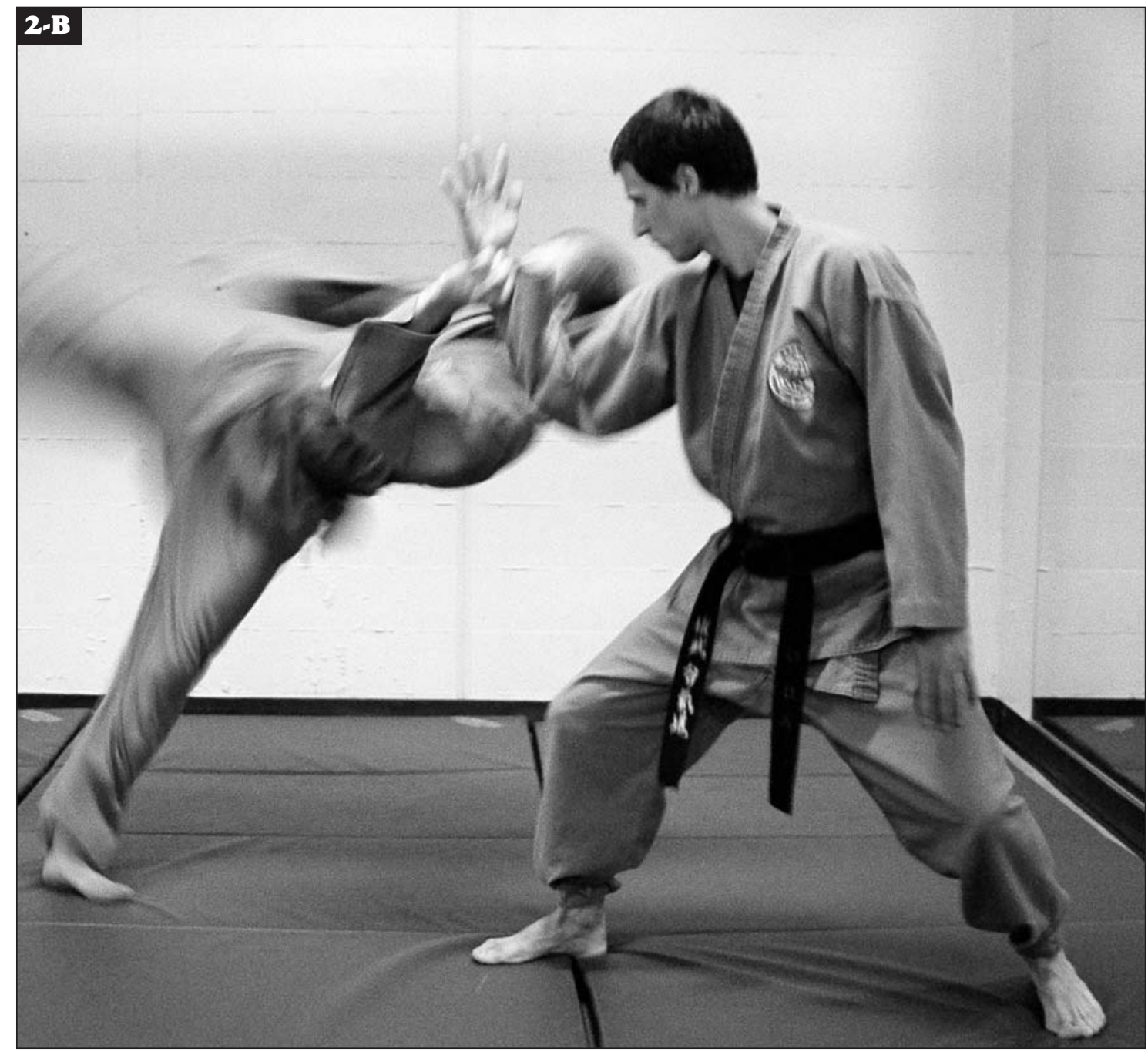




\section{Conclusión}

Aunque parezca un simple ejercicio de respiración, la DJB requiere resistencia, disciplina, y control físico y mental para realizarla adecuadamente y experimentar los beneficios de la práctica. La DJB es una técnica que necesita años de práctica para realizarse correctamente, pero los resultados valen el entrenamiento. La respiración concentrada y el movimiento del ki por la inhalación, mantenimiento, presión, y exhalación mejora la concentración mental, incrementa la fuerza física y la resistencia, y lo que es más importante, pone a punto el control del ki. Ser capaz de movilizar y cambiar de lugar el ki en tu cuerpo puede tener grandes efectos en el entrenamiento de un artista marcial así como en las actividades de cada día.

Para el entrenamiento de artes marciales, cambiar de lugar el ki puede incrementar la fuerza y el poder en ciertas técnicas. El ki puede ayudar a los músculos a trabajar más eficazmente para formar una barrera protectora contra los golpes así como para moverlo a áreas lesionadas para ayudar a facilitar la curación. En la vida cotidiana, el correcto movimiento del ki, ayuda a controlar la respiración y las funciones digestivas, lo cual puede ayudar a uno a relajarse, hacer un mejor uso del consumo de oxígeno, y ayudar en la correcta digestión de la comida.

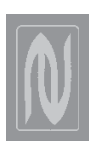

\section{Bibliografía}

Apichai, B. (2005, otoño). Qigong I. Classroom lecture. Kenmore, WA: Bastyr University.

Deadman, P., Al-Khafaji, M. \& Baker, K. (1998). A manual of acupuncture. Hove, England: Journal of Chinese Medicine Publications.

Ding, W. (2002, otoño). Traditional Chinese medicine fundamentals. Classroom lecture. Kenmore, WA: Bastyr University.

Ellis, A., Wiseman, N. \& Boss, K. (1991). Fundamentals of Chinese acupuncture. Brookline: Paradigm Publications.

Ji, Han-jae (2005, 15 de octubre). Entrevista personal. Puebla, Mexico.

Ji, Han-jae (2005, 23-25 de septiembre). Sinmoo Hapkido Seminar, Connecticut Sinmoo Hapkido. South Windsor Community Center, Connecticut.

Ji, Han-jae (2003, 24-26 de marzo). International Sinmoo Hapkido Master/ Instructor Seminar. Kenmore, WA: Bastyr University.

Ji, Han-jae (2002, 25 de diciembre). Entrevista personal. Trenton, New Jersey.

Mitchell, R. (2005, Fall). Meridians and points I. Classroom lecture. Kenmore, WA: Bastyr University.

Pak, T. (2002, 25 de diciembre). Entrevista personal. Trenton, New Jersey.

\section{Agradecimientos}

Al autor le gustaría dar las gracias al Gran Maestro Ji Han-jae y al Maestro Egil Fosslien por su ayuda y por aparecer en las fotografías utilizadas en este artículo. También manifiesta un especial agradecimiento a los siguientes estudiantes por aparecer en otras fotografías de este artículo: Yi-Pei Lin, Steve Matthewson, Jaime Drain, Tennyson Towl, Melissa Mokarzel, Lorene Ledesma, Kim Iller, Courtney Coale Carag, y Robin Terranella.

\section{Notas al pie}

${ }^{1}$ Mucha gente puede estar más familiarizada con la traducción china o pronunciación de qi. Este es el mismo concepto, pero he escogido usar la pronunciación coreana porque el Sinmoo Hapkido tiene antecedentes coreanos.

${ }^{2}$ En realidad existen seis órganos Zang y seis órganos Fu. El pericardio es el órgano Zang extra, y el Triple Calentador (San Jiao) es el órgano Fu extra. El pericardio es el recubrimiento protector exterior del corazón, y el San Jiao es la matriz de tejido conectivo que conecta los órganos internos. Están asociados con el elemento del fuego, y se suelen agrupar juntos con el corazón y el intestino delgado en la Teoría de los Cinco Elementos. 